\title{
Momentum-resolved study of an array of one-dimensional strongly phase-fluctuating Bose gases
}

\author{
N. Fabbri, ${ }^{1, *}$ D. Clément, ${ }^{1, \dagger}$ L. Fallani, ${ }^{1}$ C. Fort, ${ }^{1,2}$ and M. Inguscio ${ }^{1}$ \\ ${ }^{1}$ European Laboratory for Non linear Spectroscopy (LENS) and Dipartimento di Fisica e Astronomia, Università di Firenze, and Istituto \\ Nazionale di Ottica (INO-CNR), Sezione Sesto Fiorentino, via N. Carrara 1, I-50019 Sesto Fiorentino, Italy \\ ${ }^{2}$ Consorzio Nazionale Interuniversitario per le Scienze Fisiche della Materia UdR di Firenze, Via Sansone 1, I-50019 Sesto Fiorentino, Italy
}

(Received 17 September 2010; published 8 March 2011)

\begin{abstract}
We investigate the coherence properties of an array of one-dimensional Bose gases with short-scale phase fluctuations. The momentum distribution is measured using Bragg spectroscopy, and an effective coherence length of the whole ensemble is defined. In addition, we propose and demonstrate that time-of-flight absorption imaging can be used as a simple probe to directly measure the coherence length of one-dimensional gases in the regime where phase fluctuations are strong. This method is suitable for future studies such as investigating the effect of disorder on the phase coherence.
\end{abstract}

DOI: 10.1103/PhysRevA.83.031604

PACS number(s): 67.85.De, 05.30.Jp

Physics of one-dimensional (1D) systems attracts a great interest both on the theoretical and the experimental side. Recent progress in nanotechnology allowed the implementation of 1D systems in a variety of fields, from inorganic and organic superconductors [1] to carbon nanotubes and nanowires [2] to spin chains and ladders [3] as well as cold atomic systems [4,5]. All these systems belong to the universality class of interacting quantum fluids known as Luttinger liquids [6], whose properties strongly differ from their 3D counterparts. For instance, quantum and thermal fluctuations are strongly enhanced by the reduced dimensionality, their knowledge giving access to key quantities characterizing the system [7]. Their presence can drastically alter the properties of the systems, such as in superconductive disordered nanowires, where they can lead to the formation of phase-slip centers [8].

In the context of cold atoms, both phase and density fluctuations of 1D systems have been studied in the last years [9-12]. In particular, phase coherence has been investigated by monitoring interference between two different 1D gases [10] and by observing density modulations [11] or the response to light scattering [12] in elongated 3D quasicondensates. Nevertheless, in all these realizations, transverse trapping frequencies hardly exceed a few kilohertz and are typically of the order of the chemical potential and the temperature. Reaching the regime of strongly interacting 1D systems would further enhance the presence of quantum and thermal fluctuations. This can be achieved when atoms are loaded in 2D optical lattices, allowing for much stronger transverse confinements $[4,5]$. Yet in the latter case, one obtains a large number of 1D tubes for which techniques like [10,11] cannot be implemented to study the phase coherence properties since tube averaging washes out the response signal.

In this Rapid Communication, we investigate the axial coherence properties of an array of strongly phase-fluctuating 1D Bose gases and suggest time-of-flight (TOF) imaging as a probe of the coherence length. In our case, thermally induced phase fluctuations dominate and drastically reduce

\footnotetext{
*fabbri@lens.unifi.it

'Present address: Laboratoire Charles Fabry, Institut d'Optique, Campus Polytechnique RD128, F-91127 Palaiseau, France.
}

the coherence length $\left(2 L_{\phi}\right)$ of the system compared to a 3D Bose-Einstein condensate (BEC) [13]. We use Bragg spectroscopy with large momentum transfer [14] to measure the momentum distribution and directly evaluate $L_{\phi}$ [12]. In addition, we verify that direct mapping of the momentum distribution into coordinate space via absorbtion imaging after TOF is an effective probe of the phase fluctuations. We demonstrate that in our range of parameters, these two techniques yield the same results.

Our system, sketched in Fig. 1(a), consists of about $2 \times 10^{3} 1 \mathrm{D}$ atomic microtubes. Each gas has typical total size $\sim 30 \mu \mathrm{m} \times 0.05 \mu \mathrm{m}$ and linear density $n_{1 D} \sim 5 \mu \mathrm{m}^{-1}$. To arrange atoms in this configuration, we confine a $3 \mathrm{D}$ BEC of ${ }^{87} \mathrm{Rb}$ in a pair of orthogonal red-detuned optical lattices. We study different configurations by tuning the amplitude $V$ of the 2D optical lattice $\left(s=V / E_{R}\right.$ ranging from 5 to 56, where $E_{R}=h^{2} / 2 m \lambda_{L}^{2}, h$ being the Planck's constant, $m$ the atomic mass, and $\lambda_{L}=830 \mathrm{~nm}$ the lattice wavelength). The stronger the optical confinement, the more anisotropic is the trap experienced by each 1D gas (the aspect ratio $\lambda=\omega_{\perp} / \omega_{\|}$, namely, the ratio of the radial harmonic trapping frequency to the axial one, ranges from 787 to 880 for $5<s<56$ ). For all the amplitudes of the transverse lattices we explore, each gas has a fully 1D character, that is, both chemical potential and temperature are about 1 order of magnitude smaller than the frequency of the transverse harmonic oscillator. The crucial quantity to describe the regime of the 1D gas is the parameter $\gamma=m g_{1 D} / \hbar^{2} n_{1 D}$, that is, the ratio of interaction energy to the kinetic energy necessary to correlate particles at distance $1 / n_{1 D}, g_{1 D}$ being the interatomic coupling in the $1 \mathrm{D}$ gas. In our case, $\gamma \sim 0.3-0.6$ so that interparticle correlations are stronger than in the mean-field regime (see, e.g., [4]).

We first investigate the effect of the phase fluctuations via Bragg spectroscopy. In brief, the lattice gas is diffracted from a moving lattice created by two simultaneous off-resonant light pulses (Bragg beams) with a relative angle $\theta$, detuned from each other by a tunable frequency difference $\omega /(2 \pi)$. This perturbs the system activating excitations with energy $\hbar \omega$ and momentum $\hbar \mathbf{q}_{B}$, the modulus of which depends on $\theta$ [14]. The geometry of the Bragg beams is chosen to align $\hbar \mathbf{q}_{B}$ to the axis of the 1D tubes. After the excitation, the lattice amplitude is 


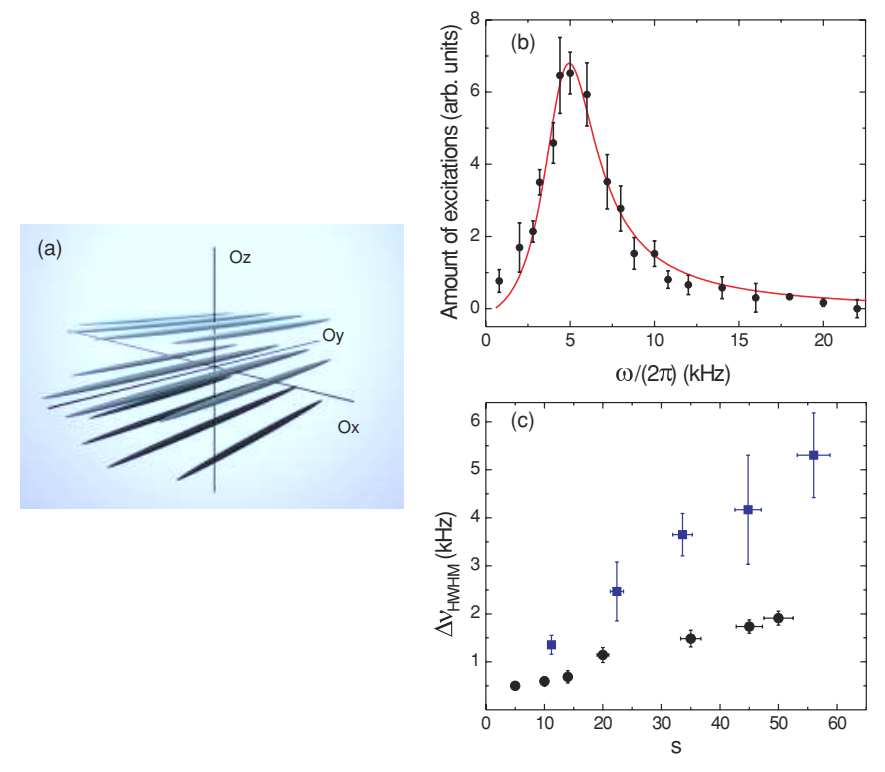

FIG. 1. (Color online) (a) Schematic view of the two-dimensional array of one-dimensional gases. (b) Bragg spectrum of an array of strongly correlated 1D gases produced by lattices with amplitude $s=50$. Red curve is a fitting of the function $v f(v)$, where $f(v)$ is a Lorentzian. (c) HWHM of the Bragg resonances as a function of the amplitude $s$ of the lattices for two different values of transferred momentum: blue squares, $q_{1}=16.0$ (2) $\mu \mathrm{m}^{-1}$; black circles, $q_{2}=$ 7.3(2) $\mu \mathrm{m}^{-1}$.

turned down in $15 \mathrm{~ms}$ to a lower value $(s=5)$, where the different tubes are no longer independent, allowing the system to rethermalize via atom-atom collisions. After $5 \mathrm{~ms}$, both optical and magnetic traps are simultaneously switched off and the system is observed after a time of flight (TOF) $t_{\mathrm{TOF}}=21 \mathrm{~ms}$. The physical observable is the increase of the size of the central peak of the atomic cloud. More detailed comments on the experimental procedure have been reported in Ref. [15]. Our technique allows us to measure the energy $\Delta E$ transferred to the system [16], which depends on the imaginary part of its polarizability $\chi^{\prime \prime}(\omega)$, apart from the characteristics of the perturbing potential (amplitude $V_{B}$ and time duration $\Delta t)$ [17]: $\Delta E \propto V_{B}^{2} \omega \chi^{\prime \prime}(\omega) \Delta t$. The polarizability can be expressed in terms of the dynamical structure factor of the system $\chi_{F}^{\prime \prime}(\omega)=\pi S(\omega, q)\left(1-e^{-\hbar \omega /\left(k_{B} T\right)}\right)$.

Two different geometrical configurations of the Bragg beams have been used to vary the transferred momentum along the axis of the gases. Counterpropagating beams along the axis of the atomic tubes yield $q_{1}=16.0(2) \mu \mathrm{m}^{-1}$; a small-angle configuration gives $q_{2}=7.3(2) \mu \mathrm{m}^{-1}$. In both cases we assume the excitation to be in the Doppler regime, ${ }^{1}$ where $S(q, \omega)$ is reduced to the momentum distribution $n(q)$ $[12,14]$. In this regime the spectral half width at half maximum (HWHM) can be related to the momentum width $\hbar \Delta q$ through the relation $\Delta v=\left(q_{B} / 2 \pi m\right) \hbar \Delta q$, which is linear in the wave vector $q_{B}$ of the excitation. ${ }^{2}$ In the experiment, the ratio

\footnotetext{
${ }^{1}$ The Doppler regime is described by the condition $q>\sqrt{4 \mu \mathrm{m}} / \hbar$, where $\mu$ is the chemical potential of the gas.

${ }^{2}$ To test the accuracy of this approximation, we calculate the HWHM of the momentum distribution for a single 1D gas from the spectral
}

of the HWHMs of the response of identical arrays of 1D gases to the two different excitations $q_{1}$ and $q_{2}$ is consistent with $q_{1} / q_{2}=(2.16 \pm 0.06)$, as expected [linear fitting of the experimental data in Fig. 1(c) allows for defining a mean ratio $\left.\Delta v_{1} / \Delta v_{2}=(2.7 \pm 0.8)\right]$.

In our range of $\gamma \approx 0.3-0.6$, interactions are beyond the mean-field description but still far from the Tonks-Girardeau regime. Thus we expect the interaction-induced spatial decay of one-particle correlation function to happen on a larger scale than that led by phase fluctuations for typical temperatures in the experiment $(\sim 100 \mathrm{nK})$ [18]. The one-particle correlation function being dominated by the exponential decay due to phase fluctuations, the momentum distribution exhibits a profile well described by a Lorentzian shape [13]. In Ref. [19], the momentum distribution for a $1 \mathrm{D}$ gas with a parabolic profile along its axis has been calculated, and the HWHM $\hbar \Delta q$ of its Lorentzian best fit has been demonstrated to rely only on the coherence length of the gas, being

$$
\Delta q=\frac{0.635}{L_{\phi}},
$$

where $L_{\phi}=\hbar^{2} n_{1 D} /\left(m k_{B} T\right)$ is the half coherence length ( $T$ being the temperature).

The description of the problem is complicated by the presence of an array of gases with different densities (and thus different characteristic $L_{\phi} \mathrm{s}$ ). In principle, one should consider the sum of the response of each tube. Supposing a mean-field picture, ${ }^{3}$ interactions would give a broadening of the width and a shift of the center of the Bragg resonance compared to the single-particle response $h v_{\mathrm{sp}}=\hbar^{2} q^{2} /(2 m)$, both depending on the density of each tube [14]. Yet the global response of the system to the Bragg excitation consists of a single broad resonance, as depicted in Fig. 1(b), the center of which is shifted compared to $v_{\mathrm{sp}}\left(v_{0}>v_{\mathrm{sp}}\right)$. Its shape is well described by $v f(v) \simeq \omega S(q, \omega)$, where $f(v)$ is a Lorentzian function [see Fig. 1(b)]. This suggests that thermal broadening of the response of each gas exceeds the interaction-induced broadening and masks the relative shifts of the resonant frequencies of the tubes. We verified numerically that this is the case for our experimental parameters [21]. Therefore we analyze the Bragg spectra as being the response of a single 1D gas, and we define accordingly an effective coherence length $L_{\phi}$ of the whole system using the relation in Eq. (1).

From the fittings of the Bragg spectra, we extract the HWHM $\Delta v$. This quantity is reported in Fig. 1(c) as a function of the amplitude $s$ of the optical lattices and for the two different wave vectors of the excitation $q_{1}, q_{2}$. The total number of atoms is kept almost constant in both the series of data. From the spectral half width, we extract the half coherence length $L_{\phi}$. As shown in Fig. 2, $L_{\phi}$ drops by a factor 5 as $s$ increases from 5 to 56 . We note that for higher $s$ values, the coherence length becomes comparable to the interparticle

width using a free-particle-like vs a Bogoliubov dispersion relation, and we verify that the results differ less than $10 \%$.

${ }^{3}$ For the values of $\gamma$ that we realize, the resonant frequencies of the Lieb-Liniger modes are indistinguishable from the mean-field solution within our experimental resolution [20]. 


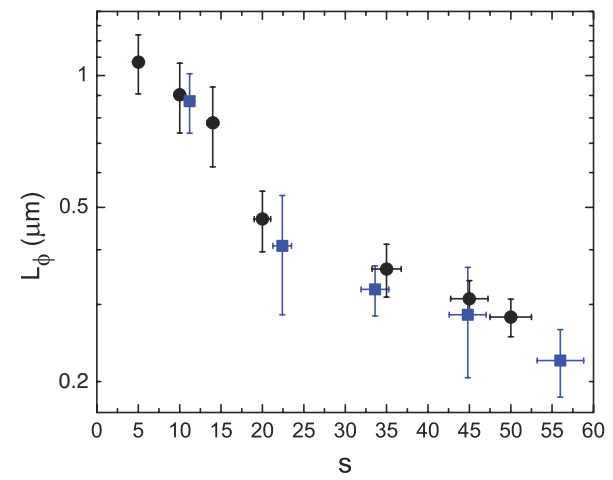

FIG. 2. (Color online) $L_{\phi}$ of the array of $1 \mathrm{D}$ gases reported as a function of the amplitude $s$ of the lattices, which squeezes the gas in 1D microtubes, and for two different values of the momentum of the excitation: blue squares, $q_{1}$; black circles, $q_{2}$.

distance. In addition, the analysis of the spectra for the two momenta $q_{1}$ and $q_{2}$ reveals consistent $L_{\phi}$, as expected in the Doppler regime. The optical confinement makes the aspect ratio of the $1 \mathrm{D}$ gases grow and their density decrease. However, we estimate numerically [21] that the relative variation of the $1 \mathrm{D}$ density in the whole range of $s$ is about $10 \%$ and does not justify the rapid downfall of the coherence length. This suggests that the major role in determining $L_{\phi}$ is played by the finite temperature. In fact, the higher the value of $s$, the larger is the axial energy spacing, and the temperature $T$ of the $1 \mathrm{D}$ gases should approximately grow proportionally. More precisely, provided that $T \gg \hbar \omega_{\|}$, as realized in our experiment $\left(\omega_{\|}\right.$ being a few tens of Hertz), the temperature is expected to be proportional to the energy $\epsilon_{j}$ of the low-energy axial modes [22], that is, in turn proportional to the frequency of the axial harmonic oscillator. This picture is indeed consistent with the experimental observation: During the adiabatic transformation of the 1D gases from $s=20$ to $s=56$, the axial energy spacing increases by a factor $\sim 1.7$; accordingly, in this range of $s$, the measured coherence length reduces by a factor $\sim 2.5$. However, note that extracting temperature from the measurement of $L_{\phi}$ is not straightforward as it requires to take into account the inhomogeneity of $n_{1 D}$ over the array; this will be the subject of a future work [21].

To keep the insight up on the system, information on the coherence length induced by thermal phase fluctuations has also been extracted by directly mapping the momentum distribution into space distribution, which is measured via absorption imaging of the gas after switching off the trap (below referred to as TOF measurements). The expansion of the atomic gas from the trap is governed by two kinds of kinetic energy: the one which interactions convert into and the one produced by in-trap phase fluctuations. Due to the strong anisotropy of the trap, the interaction-induced expansion mainly affects the radial direction [23], whereas the longitudinal size of the cloud $R_{\mathrm{TOF}}^{\mathrm{int}}$ is not significantly altered compared to its in-trap value. At nonzero temperature, thermally induced local phase gradients produce a velocity field given by $\mathbf{v}_{\phi}=(\hbar / m) \nabla \phi$ [24], $\phi$ varying significantly on a length scale $L_{\phi}$. It determines an increase $R_{\text {TOF }}^{\phi}$ of the longitudinal size during TOF, which contributes relevantly if
$R_{\mathrm{TOF}}^{\phi} / R_{\mathrm{TOF}}^{\mathrm{int}}>1$, where

$$
\frac{R_{\mathrm{TOF}}^{\phi}}{R_{\mathrm{TOF}}^{\mathrm{int}}} \sim \frac{\hbar t_{\mathrm{TOF}}}{m L_{\phi} R_{\mathrm{TOF}}^{\text {int }}} .
$$

In previous experiments, the product $L_{\phi} R_{\mathrm{TOF}}^{\text {int }}$ amounts typically to $10 \mu \mathrm{m} \times 260 \mu \mathrm{m}$ for elongated 3D quasicondensates [12] and $\sim 1 \mu \mathrm{m} \times 170 \mu \mathrm{m}$ in the case of atom-chip experiments [10]. In both cases, $R_{\mathrm{TOF}}^{\phi}$ is negligible, and the longitudinal length after TOF cannot be related to in-trap phase fluctuations. For our 1D lattice gases, this quantity is reduced to $\sim 1 \mu \mathrm{m} \times 27 \mu \mathrm{m}$ for $s=5$, and it falls even 1 order of magnitude as the amplitude of the optical confinement increases $(\sim 0.2 \mu \mathrm{m} \times 22 \mu \mathrm{m}$ for $s=56)$. This estimate refers to a representative tube with a number of atoms equal to the averaged value over all the array. Thus, in our case, one expects that the in-trap size still dominates for low values of $s$ and the density profile has a parabolic shape (possibly smoothed to a Gaussian by the finite resolution of the imaging system), as expected in the Thomas-Fermi regime. For high values of $s$, phase fluctuations should enlarge the distribution, and the profile should assume a Lorentzian shape. To confirm this behavior, we have analyzed the TOF profiles with both Gaussian and Lorentzian functions. From these fittings, we plot in Fig. 3 the mean squared value of the residuals of both fitting functions. As anticipated from the simple formula in Eq. (2), Fig. 3 points out the cross-over through the two regimes at $s \sim 10-15$. In particular, for $s>20$, initial in-trap phase fluctuations are responsible for the Lorentzian shape of the TOF momentum distribution.

To quantitatively compare the results of Bragg spectroscopy and TOF measurements, we map both energy spectra and density profiles after TOF into the momentum space of the in-trap gas. In the first case, we divide the measured amount of excitation by $\omega$, then we use the free-particle dispersion relation, obtaining $q=4 \pi^{2} m v /\left(h q_{B}\right)-q_{B} / 2$. In the latter case, the calibration of the pixel size in momentum space is obtained by measuring the distance between two interference peaks released from the lattices at weak amplitude. For an array of strongly correlated 1D gases $[s=50$ in Fig. 4(a)],

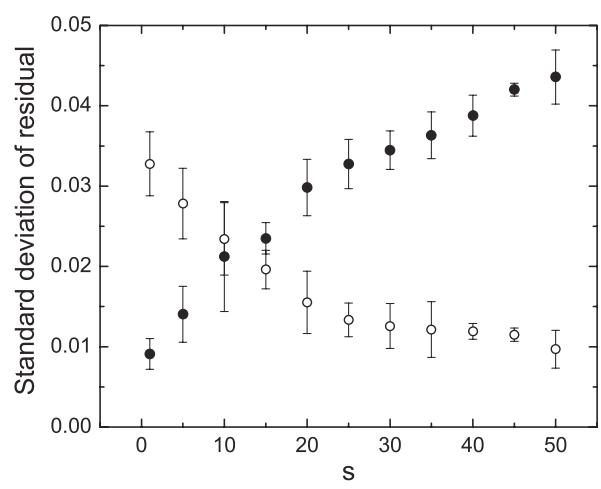

FIG. 3. Mean-square value of residuals of a Lorentzian (open circles) and Gaussian function (solid circles) fitted to the momentum distribution mapped through TOF. 



FIG. 4. (Color online) (a) Momentum distribution of an array of $1 \mathrm{D}$ gases in a strongly confining optical lattice $(s=50)$ measured through Bragg spectroscopy (black circles) and direct mapping in TOF density profile (red curve). (b) Half coherence length $L_{\phi}$ extracted from TOF measurements shown as a function of $s$ in linear scale. The gray area points out the region of parameters where TOF measurements cannot be used to extract $L_{\phi}$. Inset shows a comparison between $L_{\phi}$ from Bragg measurements (open circles) and direct mapping (red circles)

the momentum distributions measured via Bragg spectroscopy (data points) and TOF measurements (continuous line) show an excellent agreement. From TOF measurements, we extract the coherence length as well. According to our results from Bragg spectroscopy, $L_{\phi}$ is observed to reduce as $s$ increases [Fig. 4(b)]. In the inset, we compare $L_{\phi}$ measured in the two ways. The accordance fails in the region of parameters (gray area) where TOF profiles are not dominated by phase fluctuations (see Eq. (2)).

In conclusion, we investigate the coherence properties of an array of 1D Bose gases by measuring their momentum distribution. We observe the latter to have a Lorentzian shape, as predicted for a single uniform 1D gas. We define an effective coherence length of the whole ensemble, and we show its evident reduction as the optical confinement is increased. Comparing Bragg spectroscopy and direct mapping of momentum into density distribution after TOF demonstrates that TOF images give access to coherence properties in the presence of strong phase fluctuations. Our work paves the way for future studies of the coherence properties in 1D geometries with short coherence lengths. Of particular interest are strongly interacting disordered systems where the role of thermal phase fluctuations in the nature of the superconductorinsulator transition is debated [25]. So far, only disordered quasicondensates have been investigated, where it was shown that the contribution of phase fluctuations is small [26].

We thank T. Giamarchi, A. Iucci, and M. Zvonarev for fruitful discussions. This work has been supported by MIUR PRIN 2007, Ente Cassa di Risparmio di Firenze, DISQUA Project from European Research Council, and the DQS EuroQUAM project from ESF. D.C. acknowledges support of a Marie Curie Intra-European Grant.
[1] F. Wang, R. A. Cowley, S. E. Nagler, and A. M. Tsvelik, Phys. Rev. Lett. 103, 136401 (2009); J. Hager et al., ibid. 95, 186402 (2005); T. Giamarchi, Chem. Rev. 104, 5037 (2004).

[2] O. M. Auslaender et al., Science 295, 825 (2002).

[3] D. A. Tennant et al., Phys. Rev. B 52, 13368 (1995); E. Dagotto and T. M. Rice, Science 271, 5249 (1996).

[4] B. Laburthe-Tolra et al., Phys. Rev. Lett. 92, 190401 (2004).

[5] T. Kinoshita, T. Wenger, and D. Weiss, Science 305, 1125 (2004); B. Paredes et al., Nature (London) 429, 277 (2004).

[6] F. D. M. Haldane, J. Phys. C 12, 4791 (1979).

[7] T. Giamarchi, Quantum Physics in One Dimension (Oxford Science, Oxford, 2004).

[8] S. Michotte et al., Physica C (Amsterdam) 391, 369 (2003); D. Y. Vodolazov et al., Phys. Rev. Lett. 91, 157001 (2003).

[9] J. Estève et al., Phys. Rev. Lett. 96, 130403 (2006).

[10] S. Hofferberth et al., Nature (London) 449, 324 (2007).

[11] S. Dettmer et al., Phys. Rev. Lett. 87, 160406 (2001); A. Imambekov et al., Phys. Rev. A 80, 033604 (2009); S. Manz et al., ibid. 81, R031610 (2010).

[12] S. Richard et al., Phys. Rev. Lett. 91, 010405 (2003).

[13] M. A. Cazalilla, J. Phys. B 37, S1 (2004); D. S. Petrov, G. V. Shlyapnikov, and J. T. M. Walraven, Phys. Rev. Lett. 85, 3745 (2000).

[14] J. Stenger et al., Phys. Rev. Lett. 82, 4569 (1999).
[15] D. Clément et al., New J. Phys. 11, 103030 (2009).

[16] N. Fabbri et al., Phys. Rev. A 79, 043623 (2009); D. Clément, N. Fabbri, L. Fallani, C. Fort, and M. Inguscio, Phys. Rev. Lett. 102, 155301 (2009).

[17] A. Brunello, F. Dalfovo, L. Pitaevskii, S. Stringari, and F. Zambelli, Phys. Rev. A 64, 063614 (2001).

[18] V. N. Golovach, A. Minguzzi, and L. I. Glazman, Phys. Rev. A 80, 043611 (2009).

[19] F. Gerbier, Ann. Phys. Fr. 29, 1 (2004).

[20] T. Giamarchi et al. (private communications).

[21] N. Fabbri et al. (in preparation).

[22] D. S. Petrov, G. V. Shlyapnikov, and J. T. M. Walraven, Phys. Rev. Lett. 87, 050404 (2001).

[23] Y. Kagan, E. L. Surkov, and G. V. Shlyapnikov, Phys. Rev. A 54, R1753 (1996); Y. Castin and R. Dum, Phys. Rev. Lett. 77, 5315 (1996).

[24] L. Pitaevkii and S. Stringari, Bose-Einstein Condensation (Clarendon, Oxford, 2003).

[25] P. Phillips and D. Dalidovich, Science 302, 243 (2003); Y. Dubi, Y. Meir, and Y. Avishai, Nature (London) 449, 876 (2007).

[26] D. Clément, P. Bouyer, A. Aspect, and L. Sanchez-Palencia, Phys. Rev. A 77, 033631 (2008); Y. Chen et al., ibid. 77, 033632 (2008). 